\title{
Development of a Modernized Shared Laboratory for Control Systems Education
}

\author{
N. Sepehri, D. Fraser, U. Annakkage and E. Jalayeri \\ Faculty of Engineering \\ The University of Manitoba \\ Winnipeg, Manitoba, Canada R3T-5V6
}

\begin{abstract}
This paper describes an interdepartmental initiative which aimed at launching the establishment of a shared laboratory facility to support teaching courses related to control, instrumentation and Mechatronics offered by various engineering departments at the University of Manitoba. The paper first outlines the rationale and need for this initiation. The approaches taken so far from upgrading the existing equipment and making them available at the faculty level to purchasing modern equipment are described. Future steps towards using the facility to encourage problem based learning and teaching innovations will also be described.
\end{abstract}

\section{Introduction}

The concept of shared control laboratory is not new. In United States, this concept has been successfully implemented at the University of Illinois, at Urbana-Champaign $[1,2,3]$. However, to the best of our knowledge, this concept has not been implemented at any other university in Canada prior to this work. The rationale for this initiation is similar to what has been

-To whom all correspondence should be addressed: Department of Mechanical and Manufacturing Engineering.

Email: nariman@cc.umanitoba.ca documented in references [2] and [3]. Due to the need, control courses are offered by multiple departments at the University of Manitoba, as in other universities. This is necessary and desirable since it allows individual departments to retain control of their own curricula, course content, and freedom to adapt to changes in their own fields, and with respect to research activities in particular departments. However, when teaching junior level control courses, certain common benchmark experiments are often duplicated in-spite of differences in disciplines. Duplication of equipment may occur, which is undesirable from the view point of cost effectiveness. Therefore, it is beneficial, whenever possible, to combine existing control laboratory equipment to be shared by all departments. This way, current resources will be pooled, opportunity for acquiring new teaching equipment and tools will be increased, and duplication of laboratory facilities will be prevented [2].

The paper describes establishment of a shared control laboratory to support teaching core control courses offered by both the Departments of Mechanical and Manufacturing Engineering and, Electrical and Computer Engineering. To fulfill this objective, we needed to: (i) upgrade the existing equipment and make them for availability to both departments and (ii), 
purchase modernized equipment to encourage teaching advanced concepts and new technologies.

\section{Existing Laboratory Facilities}

Prior to this project, the Control and Mechatronics Laboratory at the Department of Mechanical and Manufacturing Engineering served two undergraduate core control courses required by two degree programs: "Mechanical Engineering" and "Manufacturing Engineering". The laboratory was equipped with:

(a) Four workbenches (see Fig. 1), each equipped with a Pentium computer, a DAS16 data acquisition board, a power supply, an oscilloscope, a function generator, a multimeter, a DC motor, a tachometer, a potentiometer, loading attachments and inhouse built software programs for implementation of various control algorithms and for plotting all variables.

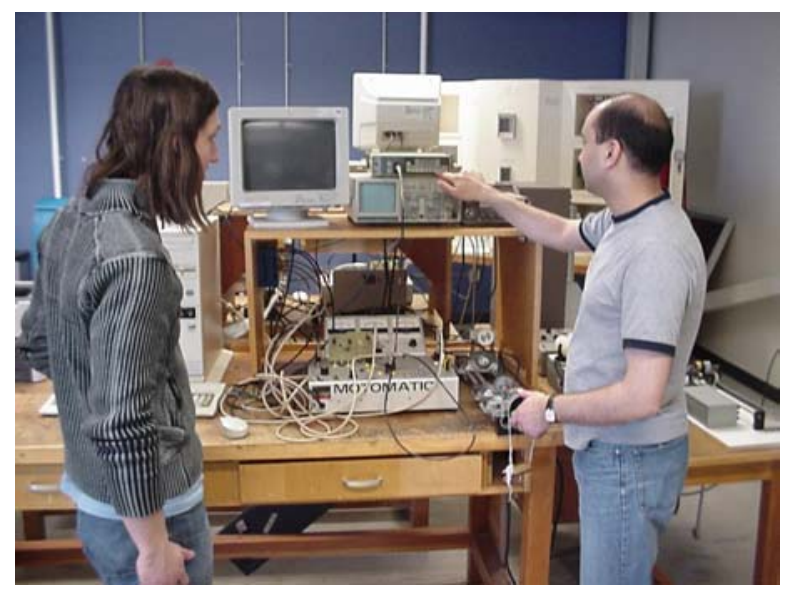

Fig. 1 Typical DC motor control workbench.

(b) An in-house built process control panel (see Fig. 2) equipped with a pneumaticallycontrolled valve, a pressure transducer, a temperature transducer and tanks for controlling pressure, temperature and water level. The panel can be controlled either by a computer through an interfacing board or a commercially available industrial controller.

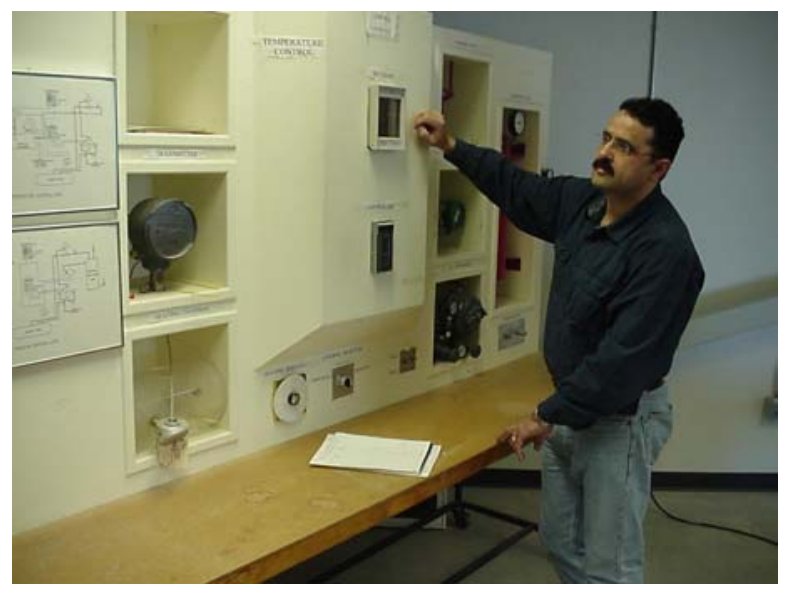

Fig. 2 Process control workstation.

(c) A conveyor belt system for event controller programming. The system is interfaced with a computer via an $\mathrm{I} / \mathrm{O}$ board and an in-house built software program.

The above facilities provided the students valuable hands-on experience with control components, allowing them to have a better understanding of theories. However, the equipment was purchased in early 1980. Since then every effort has been made to make the best use of the devices.

The control laboratory in the Department of Electrical and Computer Engineering was used to serve one undergraduate core course. The laboratory was equipped with five basic stations with analog computers for controlling motor speeds or temperature in a pipe. Each workstation was equipped with a signal generator, an oscilloscope and a multimeter. The laboratory was also used for teaching an elective advanced control course.

It is well-known that proper way of teaching control theory requires that the students be extensively involved with hands on 
experiments using fully-instrumented equipment to develop a better understanding of, and appreciate the issues involved in the area of instrumentation and control theory. The existing facilities in both departments complement each other, and could be equally used in undergraduate control courses by both departments. However, the existing facilities required upgrading. Secondly, new innovative equipment was needed to be purchased to reflect rapid changes in the technology and for teaching advanced concepts.

\section{New Laboratory Facilities}

The new shared control laboratory is now equipped with existing upgraded and functional equipment from both departments as well as many state-of-the-art new educational devices that either replace or complement the existing equipment.

(a) Basic control engineering training stations: This set of equipment (see Fig. 3) provides a simple way to demonstrate fundamentals of motor control, tuning and haptics using several integrated experiments. Each station is a portable, standalone device that is versatile and can be controlled via analog, digital, embedded or computer control. It can be connected to a PC or a laptop. The servo mechanism sets were recently used to design labs that give the students experience in the complete cycle of control systems design: modeling, model validation, analysis, controller design, rapid prototyping, implementation, and testing. The labs were highly integrated to the material taught in the class. The students learned how to model the dynamic behavior of the electrical and mechanical components of the system. They also learned how to determine some of the parameters of the model by means of static as well as response tests on the actual system. They designed PID controllers for speed control and position control, and tested the performance.

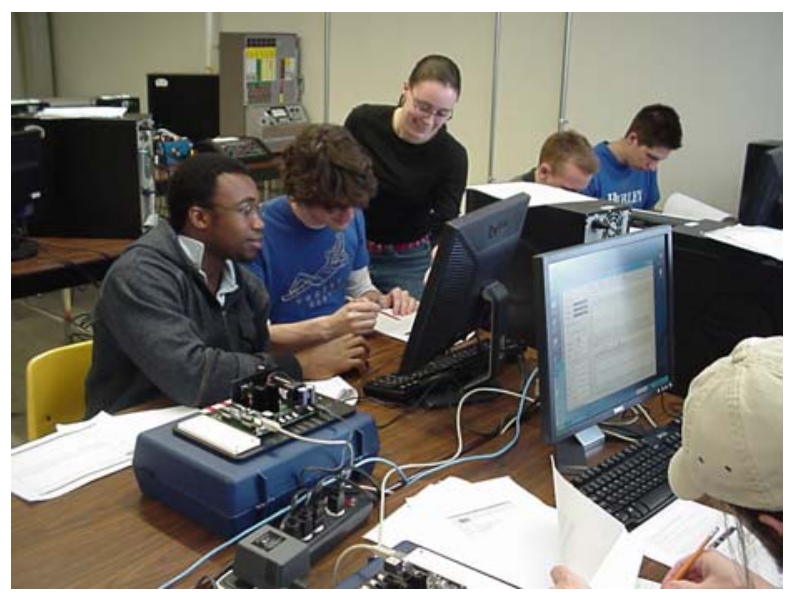

Fig. 3 Groups of $3^{\text {rd }}$ year Mechanical and Manufacturing Engineering students use the newly purchased training kits as part of the requirement for the control course.

(b) Rotary servo plants with open control architecture: This set of equipment is used to introduce fundamental control concepts, and interfacing on an easy-to-use platform. Each plant consists of a DC motor, equipped with a gearbox that drives external gears, a potentiometer, a high-resolution optical encoder, a tachometer and a data acquisition board. The system is fully compatible with MATLAB/Simulink.

(c) Ball and beam, Flexible link, Flexible joint, self erecting rotary pendulum, 2-DOF robot and 2-DOF Gantry joint pendulum modules: These modules are used with servo plants, described in (b), to provide the students with practical and real-life control problems (see Fig. 4). For example, the inverted pendulum module was used in a Control Engineering course by the Department of Electrical and Computer Engineering, to teach Linear Quadratic Regulator design. The inverted pendulum experiment was an excellent motivator for students to gain confidence in the state space 
control system design theory learned in the class. This laboratory started with the mathematical modeling of the inverted pendulum. Students were given the basic equations that govern the dynamic behavior of the pendulum. They had an exposure to nonlinear control systems and their linearization during the lectures. The laboratory helped the students reinforce their understanding of nonlinear systems. In this laboratory, they established acceptable ranges for state variables for the linearized model to be a good representation of the nonlinear system.

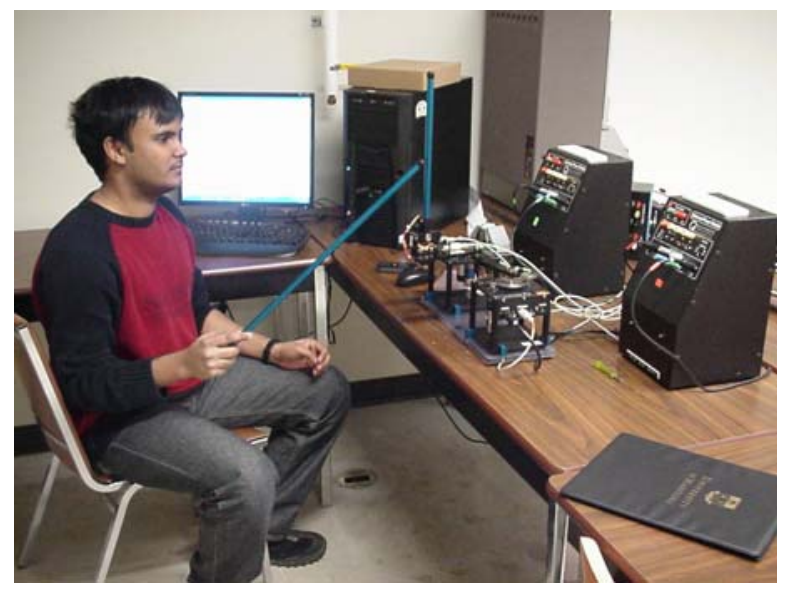

Fig. $44^{\text {th }}$ year Mechanical Engineering student is conducting a test on the 2-DOF inverted pendulum module as part of his thesis project.

(d) QIC Mechatronics: The device allows for the quick implementation of a complete control. Using a built in programmer one can quickly download compiled codes to the PIC. The device can readily implement controllers ranging from pure state machines to complex MIMO state feedback digital controllers.

(e) High fidelity linear cart system (HFLC): This equipment is meant for advanced courses. The HFLC consists of a cart equipped with a 400 Watt motor. The motor is equipped with a pinion that drives against a toothed rack such that the cart moves in a linear fashion. The cart is equipped with two un-powered joints instrumented with optical encoders. These joints can be used to mount up to two rotary pendulums directly on the cart to perform a variety of experiments: linear speed control, linear position control, single inverted pendulum, self erecting single pendulum, dual inverted pendulum, double inverted pendulum, triple inverted pendulum, single pendulum gantry, double pendulum gantry and triple pendulum gantry.

The newly purchased devices were chosen to provide the students with variety of experiments. Some of the devices are portable and can be easily taken home and connected to laptops by students to perform their laboratory experiments. Other devices consist of modular actuators, sensors, interfacing boards and other accessories in order to establish a general platform for the students to design, built and test their ideas as solutions to problems originating from reallife automation scenarios. Balance between theory, analysis and hardware implementation has been emphasized in the new laboratory setup. Modeling, analysis, control techniques, computer simulations, hardware implementation and comparison between simulations and experiments are now possible in the shared laboratory facility. Additionally, with the new devices, the students will be able to design advanced control design techniques and understand the digital implementation of control schemes, real-time programming and interfacing issues.

All control courses in both the Departments of Mechanical and Manufacturing Engineering and, Electrical and Computer Engineering, are now utilizing the equipment in a newly-assigned laboratory space. However, the two departments will retain 
control over their specific courses and are responsible for providing their own TAs. The day-to-day activities of the labs are planned to be managed by one or two lab specialists who will closely work with the academics in charge of teaching the core control courses.

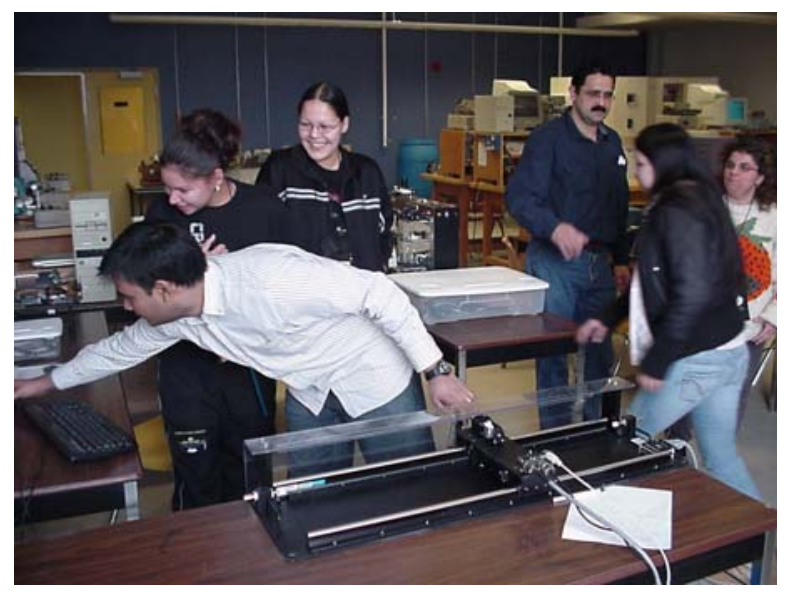

Fig. 5 As part of promoting engineering to high school students, the laboratory hosted few high school students to participate in an experiment with HFLC system.

\section{Significance}

(a) The development of a shared control laboratory to support courses related to control systems in the faculty of engineering, will further enhance engineering education at the University of Manitoba. This is in-line with our continuous effort to promote and upgrade the level of education at our university and at the same time, improve the curriculum to meet the requirement for future engineers. To the best of our knowledge, the concept of shared control laboratory has not been implemented at any other university in Canada.

(b) The collaboration between the two major departments, and the experience and knowledge obtained, through implementation of this project has brought professors involved in teaching and research in the area of control closer. This will further lead to future initiatives, which will enrich the entire control engineering (both undergraduate and graduate) program at our university. For example it could lead to:

(i) Development of highly interdisciplinary project-based learning setting to teach concepts in control systems. Projectbased learning is one of the latest and most innovative undergraduate education methodologies that partially replaces traditional lectures with group learning via working on state-of-the-art practical applications.

(ii) Design of joint undergraduate thesis projects that can later be utilized as undergraduate teaching tools.

(iii) Organization of short-term specialized control courses to participants from industry through continuing education.

\section{Discussion on Future Steps}

Presently, we study the introduction of problem-based projects in teaching control courses. In a project-based learning setting, the students will be exposed to credible and more challenging control problems and learn as they need. This way the students will gain greater technical skills sooner and become technically more mature.

The distinction between project-based learning and other forms of co-operative or active learning often are blurred because they share certain common features. An essential component of problem-based learning is that the problem represents complex real-world problems. This contrasts with common teaching strategies where the concepts, presented in a lecture format, precede "endof-the-chapter" problems. Students working in small groups, identify what they know, and more importantly, what they don't know. They must go beyond their textbooks. The 
primary role of the instructors is to facilitate group process learning and provide direction and not to provide easy answers. Weaker students may find this most frustrating while most of the stronger students find it a great challenge. Other benefits are as follows: (i) students learn and practice skills in problem solving, communication and selfmanagement, (ii) positive communication and collaborative relationships among diverse groups of students are created, (iii) bored or indifferent students are engaged and motivated easier and (iv), students will have the opportunity to teach faculties, which improves their own confidence and selfesteem.

\section{Conclusions}

This paper briefly described development of a shared laboratory for control systems educations at the University of Manitoba. The laboratory has been designed to provide the students with variety of experiments. Some of the devices are portable and thus can be easily taken home by students to perform their laboratory experiments. Other devices are modular in order to establish a general platform for the students to design, built and test their own ideas in a project-based setting. The new laboratory allows the students to design advanced techniques and understand the digital implementation of control, realtime programming and interfacing issues.

The lead author (N. Sepehri) would welcome inquiries and be pleased to furnish more information to faculty members of other institutions.

\section{Acknowledgements}

The authors wish to thank Dr. Ruth, Dean of the Faculty of Engineering, for his support and the University of Manitoba Engineering Endowment Fund Committee for providing the funding. The authors also wish to acknowledge the contributions of many undergraduate and graduate students involved in setting up and testing the new equipment.

\section{References}

[1] Spong, M.W., "Undergraduate Research in Robotics and Automation at the University of Illinois at Urbana-Champaign," 35th IEEE CDC, Kobe, Japan, 1996.

[2] Alleyne, et. al., A College-wide LaboratoryBased Program in Control Systems Technology at the University of Illinois at Urbana Champaign," $35^{\text {th }}$ IEEE CDC, Kobe, Japan, 1996.

[3] Spong M.W., "Control Education Crossing Department Boundaries", American Control Conference, San Diego, CA, 1999. 